\title{
Plasma heating and ultrafast semiconductor laser modulation through a terahertz heating field
}

\author{
Jianzhong $\mathrm{Li}^{*}$ and C. Z. Ning ${ }^{\dagger}$ \\ NASA Ames Research Center, M/S N229-1, Moffett Field, CA94035-1000
}

(May 24, 2000)

\begin{abstract}
Electron-hole plasma heating and ultrafast modulation in a semiconductor laser under a terahertz electrical field are investigated using a set of hydrodynamic equations derived from the semiconductor Bloch equations. The selfconsistent treatment of lasing and heating processes leads to the prediction of a strong saturation and degradation of modulation depth even at moderate terahertz field intensity. This saturation places a severe limit to bandwidth achievable with such scheme in ultrafast modulation. Strategies for increasing modulation depth are discussed.
\end{abstract}

"jianzhng(r) nas.nasa.gov

†'ning(1) naks.natsa.gov 


\section{INTRODUCTION}

As demand keeps increasing for faster data transfer rate in torlay's broalband telecommunication systems, conventional current injection modulation scheme on semiconductor diode laser and passive modulation schemes, such as those using electro-optic and acousticoptic effects, are stretched to their limits. ${ }^{1}$ As a result, both multiplexing technologies and alternative modulation schemes into the terahertz $(\mathrm{THz})$ regime are under fervent pursuit. Direct optical modulation of the transmitter laser is distinctively advantageous over other passive schemes as the telecommunication industry is moving toward an all-optical networking future in order to eliminate the electronic bottleneck. One of such alternative direct modulation schemes is through a heating electrical field..$^{2-5}$

In such a modulation scheme for quantum well lasers, a high frequency electrical field $(\mathrm{HFF})^{2,3}$ or a terahertz field (THF $)^{4,5}$ is applied to the laser structure such that the field polarization is in the plane of quantum well layers. The applied field accelerates electronhole plasma and the acquired kinetic energy is then converted to thermal energy through various scatterings to heat the plasma. For a time-dependent field, the plasma temperature is expected to change, following the time-dependence of the applied field. Since it is well-known that optical gain is a sensitive function of the electron-hole plasma (EHP) temperature, the alternating plasma temperature will lead to an alternating optical gain, thus to a modulated laser output. In contrast to the conventional current injection modulation, where the bandwidth ${ }^{6}, f_{3 d B}$, is limited by interband recombination carrier lifetime $\tau_{c}$ and density-induced differential gain $\partial G / \partial N$, modulation through a $\mathrm{THz}$ field should not experience this speed bottleneck, since plasma heating is an intraband process which occur on picosecond time scale. The plasma density is almost constant on such a fast time scale, so that slow recombination is no longer an issue in this scheme. This is the basic idea behind the ultrafast laser modulation through a high frequency heating field.

The basie phenomena of this modulation scheme have been discussed in several previous paperse ${ }^{2,3,5}$ using simplified models. In those approaches, linearization of the optical gatin at a 
given temperature is made. The self-consistent coupling between lasing and plasma heating processes is not fully considered. One obvious prediction of such a theory, though not, explicitly emphasized in those papers, is that the modulation depth increases monotonously as a function of the THF/HFF amplitude. One is led to believe that the desired depth of modulation can always be achieved through an increase of the amplitude of the heating field. Since modulation depth is an important parameter for any practical implementation of this scheme, it is important to look at this issue more closely to find out if and to what degree the prediction of a simplified linear theory is valid.

In this paper, we attempt to overcome these shortcomings of the linear theory and to address the two-way coupling between lasing and heating processes. While effects of plasma heating on lasing process has been addressed, the reverse process, namely the effect of lasing on heating has not been adequately studied. Our starting point is the well-established semiconductor Bloch equations (SBEs) that describe microscopically the coupling between plasma distribution and optical polarization induced by the laser field in the active semiconductors. We derive a set of equations for moments of up to second order from the SBEs after adiabatically eliminating the medium polarization. This set of moment equations consists of energy, momentum, and carrier density. Their self-consistent coupling and the microscopic gain are included automatically.

This paper is organized as follows: In the next section, we will derive the set of moment equations from the SBEs and review the basic assumptions we make in the process of derivation. In the third section, main results of our numerical simulation of these moment equations will be presented. Finally, the paper is concluded in the fourth section.

\section{HYDRODYNAMIC MODEL: THE MOMENT-EQUATION METHOD}

The physical system we are dealing with is a uniform EHP in a semiconductor quantum well interacting with a single-mode laser field by stimulated (mission and absorption, which is conveniently described by the two-band semienductor Bloch equations. ${ }^{7}{ }^{9}$ The modified 
SBEs ${ }^{10} 12$ with an in-plane terahertz field in the direction of $\hat{x}, \vec{F}_{T H z}(t)=F_{T H z}(t) \hat{x}$, are used as our starting point to derive the moment equations and listed below,

$$
\begin{aligned}
& {\left[\frac{\partial}{\partial t}-\frac{e \vec{F}_{T H z}(t)}{\hbar} \cdot \nabla_{\vec{k}}\right] n_{e}(\vec{k})=-2 \Im\left\{\Omega_{\vec{k}} p_{\vec{k}}^{*}\right\}+\left.\frac{\partial n_{e}(\vec{k})}{\partial t}\right|_{i n c}} \\
& {\left[\frac{\partial}{\partial t}-\frac{e \vec{F}_{T H z}(t)}{\hbar} \cdot \nabla_{\vec{k}}\right] n_{h}(-\vec{k})=-2 \Im\left\{\Omega_{\vec{k}} p_{\vec{k}}^{*}\right\}+\left.\frac{\partial n_{h}(-\vec{k})}{\partial t}\right|_{i n c}} \\
& {\left[i \hbar\left(\frac{\partial}{\partial t}-\frac{e \vec{F}_{T H z}(t)}{\hbar} \cdot \nabla_{\vec{k}}\right)+\left(\hbar \omega_{l}-\varepsilon_{e}(\vec{k})-\varepsilon_{h}(\vec{k})-E_{g}\right)\right] p_{\vec{k}}} \\
& =\hbar \Omega_{\vec{k}}\left[n_{e}(\vec{k})+n_{h}(\vec{k})-1\right]+\left.i \hbar \frac{\partial p_{\vec{k}}}{\partial t}\right|_{i n c}
\end{aligned}
$$

where $n_{e, h}( \pm \vec{k})$ is the nonequilibrium distribution function for electrons and holes with wavevector $\pm \vec{k}$, respectively. The sign $\Im$ means the imaginary part of the complex quantity within the ensuing braces and the superscript symbol ${ }^{*}$ means complex conjugate. The $\Omega_{k}$ is the renormalized Rabi frequency and defined through

$$
\hbar \Omega_{\vec{k}} \equiv d_{c v}(\vec{k}) A+\sum_{\vec{k}^{\prime} \neq \vec{k}} V_{\vec{k}^{\prime}-\vec{k}} p_{\vec{k}^{\prime}}
$$

Denoted by $A$ and $p_{\vec{k}}$ are the slowly-varying envelop functions of the laser field, $E(t)=A \exp \left(i \omega_{l} t\right)+c . c$, and interband optical polarization, $P_{\vec{k}}(t)=p_{\vec{k}} \exp \left(i \omega_{l} t\right)$. For brevity, most notation of the time dependence has been dropped. As usual, $e$ is the absolute value of electron charge, $\hbar$ is the Planck's constant, $V_{\vec{q}}$ is the Fourier component of the screened Coulomb interaction between an electron and a hole, $d_{c v}(\vec{k})$ is the interband optical dipole matrix element between an electron state, $|c \vec{k}\rangle$, and a hole state, $|v-\vec{k}\rangle, \varepsilon_{e, h}(\vec{k})$ is the carrier energy renormalized by the screened Coulomb interaction, $\hbar \omega_{l}$ is the photon energy of the laser field, and $E_{g}$ is the bandgap energy of the semiconductor gain medium. Major incoherent scattering processes, such as carrier-carrier and carrier-LO phonon collisions, are lumped in the terms labeled by subscript inc and treated in the second-order Born approximation. ${ }^{13,9}$ Last but not least, other contributions not included here will be added empirically to the moment equations, as appeared in Eqs. (2.7-2.9). Before delving into further development of the above nquations, it is helpful to discuss om treatment of the scattring processes. In 
this paper, temporal correlations are assumed to be instantaneous for the statistical evolution of the EHP. In the semiclassical picture, by neglecting memory effect, the incoherent scattering terms in the modified SBEs are expressed in terms of local carrier distributions,

$$
\begin{aligned}
\left.\frac{\partial n_{\alpha}(\vec{k})}{\partial t}\right|_{i n c} & =\frac{2}{S} \sum_{\vec{k}^{\prime}}\left\{W_{\alpha}\left(\vec{k}, \vec{k}^{\prime}\right) n_{\alpha}\left(\vec{k}^{\prime}\right)\left[1-n_{\alpha}(\vec{k})\right]-W_{\alpha}\left(\vec{k}^{\prime}, \vec{k}\right) n_{\alpha}(\vec{k})\left[1-n_{\alpha}\left(\vec{k}^{\prime}\right)\right]\right\} \\
\left.\frac{\partial p_{\vec{k}}}{\partial t}\right|_{\text {inc }} & =\frac{2}{S} \sum_{\vec{k}^{\prime}}\left[W_{p}\left(\vec{k}, \vec{k}^{\prime}\right) p_{\vec{k}^{\prime}}-W_{p}\left(\vec{k}^{\prime}, \vec{k}\right) p_{\vec{k}}\right]
\end{aligned}
$$

where $\alpha=e, h$, shorthands for electrons and holes, $S$ is the active area of the QW. The Boltzmann scattering matrices $W_{\alpha}\left(\vec{k}, \vec{k}^{\prime}\right)$ for electrons and holes and the scattering matrices $W_{p}\left(\vec{k}, \vec{k}^{\prime}\right)$ take into account of carrier-carrier and carrier-phonon scattering processes additively (see Ref. 14 for detail).

It is computationally too expensive and very often unnecessary to solve the above $k$ resolved equations to describe a laser. Rather a set of moment equations is simpler and often used. This has been done for lasers without the heating field ${ }^{15-18}$, where the equation for the first-order moment, the momentum, was not necessary. Formally it is straightforward to write down equations for the various moments. For the first three moments, we have the following equations (see Ref. 19 for a general approach):

$$
\begin{aligned}
& \frac{d N}{d t}=-\frac{4}{S} \sum_{\vec{k}} \Im\left\{\Omega_{\vec{k}} p_{\vec{k}}^{*}\right\}|A|^{2}+\frac{\eta J_{i n j}}{e}-\frac{B_{s p}}{w} N^{2} \dot{-} \gamma_{n r} N \\
& \frac{d P_{\alpha}}{d t} \pm e N F_{T H z}(t)=-\frac{4}{S} \sum_{\vec{k}} \hbar k_{x} \Im\left\{\Omega_{\vec{k}} p_{\vec{k}}^{*}\right\}|A|^{2}+\left.\frac{d P_{\alpha}}{d t}\right|_{\alpha \beta} \\
& +\left.\frac{d P_{\alpha}}{d t}\right|_{c-L O}-\frac{B_{s p}}{w} N P_{\alpha}-\gamma_{n r} P_{\alpha} \\
& \frac{\dot{d} E_{\alpha}}{d t} \pm e \frac{P_{\alpha}}{m_{\alpha}} F_{T H z}(t)=-\frac{4}{S} \sum_{\vec{k}} \frac{\hbar^{2} k^{2}}{2 m_{r}} \Im\left\{\Omega_{\bar{k}} p_{\vec{k}}^{*}\right\}|A|^{2}+\left.\frac{d E_{\alpha}}{d t}\right|_{\alpha \beta} \\
& +\left.\frac{d E_{\alpha}}{d t}\right|_{c-L O}+\frac{\eta J_{i n j}}{e}\left(\nu E_{g}+\frac{E_{\alpha}}{N}\right)-\frac{B_{s, j}}{w} N E_{\alpha}-\gamma_{n r} E_{\alpha}
\end{aligned}
$$

where

$$
\left\{N_{\alpha}, P_{\alpha}, E_{\alpha}\right\}=\frac{2}{S} \sum_{\vec{k}}\left\{1, \hbar k_{r}, \frac{\hbar^{2} k^{2}}{2 m_{c k}}\right\} n_{\alpha}(\vec{k})
$$

u' is the quantum well width, and $m_{r}=m_{r} m_{h} /\left(m_{r}+m_{h}\right)$ is the reduced mass. The $\hat{y}^{-}$ component of the momentum $\vec{P}_{a}$ has no dynamical relevance in our case and thus been 
left out so $P_{\alpha}$ stands for the $\hat{x}$-component. The upper/lower sign in Eqs. $(2.8,2.9)$ is for clectrons and holes, respectively. Uniformity, or charge neutrality, of the system requires $N_{e}=N_{h}=N . J_{i n j}$ is injection current with quantum efficiency $\eta$. Details in carrier capture are not considered, but we assume that the capture process occurs instantaneously and pairwise for electrons and holes. Energy gain per pair is intuitively taken as the bandgap offset $\Delta E_{g}$ between barrier and well material, in addition to the average energy $E_{\alpha} / N$ per pair injected into the EHP.

It is worth noting that other than the introduction of those empirical terms accounting for carrier generation and recombination, no assumption has been made about the distribution functions up to this point. The collision terms, $d\left\{P_{\alpha}, E_{\alpha}\right\} /\left.d t\right|_{\alpha \beta}$, for electron-hole scattering, and $d\left\{P_{\alpha}, E_{\alpha}\right\} /\left.d t\right|_{c-L O}$, for carrier-LO phonon scattering, are treated in the Born approximation. ${ }^{13,9}$ Their corresponding expressions can be easily obtained by multiplying $\hbar k_{x}$ or $\hbar^{2} k^{2} /\left(2 m_{\alpha}\right)$ to the scattering terms and then integrating over $\vec{k}$. For example,

$$
\left.\left.\frac{d\left\{P_{\alpha}, E_{\alpha}\right\}}{d t}\right|_{\alpha \beta} \equiv \frac{2}{S} \sum_{\vec{k}}\left\{\hbar k_{x}, \frac{\hbar^{2} k^{2}}{2 m_{\alpha}}\right\} \frac{\partial n_{\alpha}(\vec{k})}{\partial t}\right|_{\alpha \beta} .
$$

It is important to point out that the above set of equations is not closed yet. First, the distribution functions appear in the collision terms, Eqs. $(2.5,2.6)$. Assumption has to be made about the type and nature of these functions so that those collision terms can be evaluated. Second, the $k$-resolved polarization functions appear in the moment equations, Eqs. (2.7-2.9), which is an additional coupling that is not found in the typical moment equation treatment of electronic transport problems. This is the well-recognized truncation problem.

Our way of truncating this hierarchy relies on the time-scale argument for the problem we deal with here. We note that the fastest time scale is the carrier-carrier scatterings which complete around $50 \sim 100 \mathrm{fs}$ at the level of lasing densities. The next fastest time scale is that of the carricr-phonon scatterings, which is in the order of a few hundred fs. For the teraherta fichl we consider here, the period is larger that the phonon seattering time. Since ont main interest in this paper is on the ferahertz heating and much slower lasing 
processes, we can safely assume that the carrier-carrier scattering processes have resulted in quasi-steady distributions for clectrons and holes, or quasiequilibrium. This leads in general to the so-called drifted Fermi-Dirac distribution:

$$
n_{\alpha}(\vec{k})=f_{\alpha}^{(0)}\left(\vec{k}-\vec{k}_{D}^{(\alpha)}\right)=\left\{\exp \left[\beta\left(\epsilon_{\alpha}\left(\vec{k}-\vec{k}_{D}^{(\alpha)}\right)-\mu_{\alpha}\right)\right]+1\right\}^{-1}
$$

with $\hbar \vec{k}_{D}^{(\alpha)}=\hbar k_{D}^{(\alpha)} \hat{x}$ being the drift momentum of electrons/holes due to the applied THF/HHF in the $\hat{x}$ direction. And $\beta=\left(k_{B} T\right)^{-1}, k_{B}$ is the Boltzmann constant, $T$ is the plasma temperature, $\epsilon_{\alpha}(\vec{k})$ is the unrenormalized carrier energy, and $\mu_{\alpha}$ is the chemical potential which is determined independently for electrons and holes from their density and temperature. ${ }^{8}$ In doing so, the active semiconductor QW medium is characterized by plasma density, temperature, and the drift momenta of electrons and holes, respectively. Here we have assumed that the plasma is thermalized by the subpicosecond incoherent scatterings such that no temperature difference exists between electrons and holes (see the Appendix). The assumption about the distribution functions cut off the hierarchy arising from the scattering terms. The same time scale argument also helps cut off the hierarchy due to polarization, since distribution thermalization and polarization relaxation are the results of the same carrier scatterings. This leads to a typical adiabatic elimination of the polarization. After defining $p_{\vec{k}}=\epsilon_{0} \chi_{\vec{k}} A$ and making a relaxation time approximation for the interband polarization scattering term in Eq. (2.3),

$$
\left.\frac{\partial p_{\vec{k}}}{\partial t}\right|_{i n c} \approx-\gamma_{p} p_{\vec{k}}
$$

the $k$-resolved optical susceptibility is written as

$$
\chi_{\vec{k}}=\frac{d_{c v}(\vec{k})\left[f_{e}^{(0)}(\vec{k})+f_{h}^{(0)}(\vec{k})-1\right]}{\hbar \omega_{l}-\varepsilon_{e}(\vec{k})-\varepsilon_{h}(\vec{k})-E_{g}+i \hbar \gamma_{p}} \times \frac{1}{1-q_{1}(\vec{k})}
$$

where the term $1 /\left(1-q_{1}(\vec{k})\right)$ is the so-called Coulomb enhancement factor and its explicit expression can be found in Heug and Korh, ${ }^{8}$ or in Chow and Koch ${ }^{20}$ for the diagonal decay, or an improved version in Ning et. al. ${ }^{15}$ including the non-diagonal scatterings. The relaxation rate $\gamma_{p}$ is assumed to be $k$-independent. The many-body effects is treated in the franework 
of the Hatree-Fock theory with dynamic sereening modeled by the single plasmon pole approximation. ${ }^{78}$ It is worth noting the followings: (1) As discussed in the Introduction, subpicosecond incoherent scatterings allow us to neglect the coherent effects of the THz finld on the polarization; (2) drift-related correction to the susceptibility has been omitted for the drift kinetic energy is estimated two orders of magnitude smaller than the chemical potential at a typical THF of a few $\mathrm{KV} / \mathrm{cm}$; (3) No spectral hole burning introduced saturation effects are considered in the optical susceptibility because the laser field intensity involved in our simulation is much smaller than the required intensity, estimated at $58 \mathrm{MW} / \mathrm{cm}^{2} .^{21}$

If we substitute Eqs. (2.14) and (2.12) into the moment equations, we have a closed set of equations for the first three moments. This set, coupled with Maxwell equation, will permit a self-consistent description of lasing and heating due to the terahertz field. However, further simplification can be made to eliminate the momentum equations. First, we point out that in Eq. (2.8) the momentum change due to stimulated emission can be ignored in comparison with those due to terahertz field and to carrier-carrier and carrier-phonon scatterings. The momentum equation is then reduced to

$$
\frac{d P_{\alpha}}{d t} \pm e N F_{T H z}(t)=-\gamma_{t}^{\alpha} P_{\alpha} \mp \gamma_{e h} m_{r}\left(\frac{P_{e}}{m_{e}}-\frac{P_{h}}{m_{h}}\right)
$$

where $\gamma_{t}^{\alpha}$ is the lumped carrier momentum relaxation rate due to LO phonon scatterings, spontaneous emissions, and non-radiative recombinations, $\gamma_{e h}$ is the interband electron-hole scattering rate, which is given in the Appendix. Manipulation of this equation by Fourier transformation produces the following results,

$$
P_{e}(\omega)=-P_{h}(\omega)=\frac{e N F_{T H z}(\omega)}{\gamma_{t}+\gamma_{e h}-i \omega}
$$

Since the $\mathrm{TH} z$ frequency $\omega$ is an order of magnitude smaller than $\gamma_{t}+\gamma_{e h},{ }^{22-25}$ the frequency dependence is omitted in our investigation.

The final procedure in arriving at the hydrodynamic model is to convert the energy rquation, Eq. (2.9), to a temperature cquation, which is aided by first decomposing energy. $E_{\alpha r}$ into kinctic energy which is associated with drift momentum $P_{\alpha}$ and thermal energy If by using the following relation, 


$$
E_{\alpha}=\frac{P_{\alpha}^{2}}{2 N m_{\alpha}}+W_{\alpha}
$$

Then, the desired moment equations for the hydrodynamic model for plasma density $N$ (zeroth-order moment) and total thermal energy $W^{*}=W_{e}+W_{h}$ (second-order moment), coupled to the laser-field amplitude-envelop-function equation, ${ }^{26}$ are derived as follows,

$$
\begin{gathered}
\frac{d \mathcal{E}}{d t}=-\frac{v_{g} K \Gamma}{2 \epsilon_{0} \epsilon_{b}} \Im\left\{\frac{2}{V} \sum_{\vec{k}} d_{c v}(\vec{k})_{\chi_{\vec{k}}}\right\} \mathcal{E}-\frac{\mathcal{E}}{\tau_{p h}} \\
\frac{d N}{d t}=-\frac{4}{\overline{\hbar S}} \sum_{\vec{k}} \Im\left\{d_{c v}(\vec{k}) \chi_{\vec{k}}^{*}\right\} \mathcal{E}^{2}+\frac{\eta J_{i_{n j}}}{e}-\frac{B_{s p}}{w} N^{2}-\gamma_{n r} N \\
\frac{d W}{d t}=-\frac{4}{\hbar S} \sum_{\vec{k}} \frac{h^{2} k^{2}}{2 m_{r}} \Im\left\{\left[d_{c v}(\vec{k})+\sum_{\vec{k}^{\prime}} V_{\vec{k}^{\prime}-\vec{k}} \chi_{\vec{k}^{\prime}}\right] \chi_{\vec{k}}^{*}\right\} \mathcal{E}^{2}+\left.\frac{d W}{d t}\right|_{c-L O} \\
+\frac{\eta J_{i n j}}{e}\left(\Delta E_{g}+\frac{W}{N}\right)+\frac{N e^{2} \tau_{r H}=}{m_{r}} F_{T H z}^{2}(t)-\frac{B_{s p}}{w} N W-\gamma_{n r} W .
\end{gathered}
$$

where $\mathcal{E}=|A|, K=\sqrt{\epsilon_{b}} \omega_{l} / c$ is the wavenumber of the single-mode laser field, $c$ is the speed of light in vacuum, and $V=w \times S$ is the volume of the active region. In addition, $\tau_{T H z}=1 /\left(\gamma_{t}+\gamma_{e h}\right)$ is an effective relaxation time, similar to that in the Drude model for a single-component plasma. The weak energy dependence of both $\gamma_{t}$ and $\gamma_{e h}$ has been neglected in our model, so a constant relaxation time has been used in this paper. Carrier-LO phonon interactions, $\left.\frac{d W}{d t}\right|_{c-L O}$, are treated microscopically and details are given elsewhere. ${ }^{27}$

Rather than solving the above set of equations for $\{\mathcal{E}, N, W\}$ with the plasma temperature $T$ as a dummy variable, it is more convenient to work with the temperature and numerically solve for $\{\mathcal{E}, N, T\}$. A transformation is introduced below, which is equivalent to a similar manipulation, ${ }^{16}$ based on the chain rule and the functional relationship, $W=W(N, T)$,

$$
\frac{d T}{d t}=\left(\frac{d W}{d t}-\frac{\partial W}{\partial N} \times \frac{d N}{d t}\right)\left(\frac{\partial W}{\partial T}\right)^{-1},
$$

where $\partial W / \partial N$ and $\partial W / \partial T$ are calculated using the assumed quasi-equilibrium Fermi-Dirac distribution functions for electrons and holes.

\section{NUMERICAL RESULTS AND DISCUSSIONS}

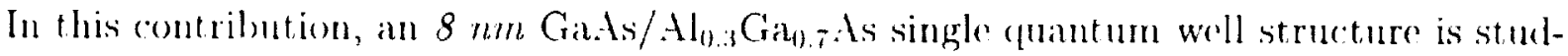
ind. Other than those material parameters, which can be easily found in literature and not 
listed here, we use the parameters in Table I. Throughout this study, we assume, except when specified otherwise, a fixed lattice temperature of $295 \mathrm{~K}$ and photon energy, $\hbar \omega_{l}$, of $1.4 \mathrm{eV}$, which roughly corresponds to the gain paik photon energy. In addition, continuous-wave (CW) THz field of the form, $F_{T H z}(t)=A_{T H z} \sin \left(2 \pi \nu_{T H z} t-\phi_{0}\right)$, is assumed. The focus of this paper is on long-time behavior of the semiconductor laser under such $\mathrm{CW}$ terahertz field. The transient response and the effects of pulsing terahertz field will be considered in a forthcoming paper.

As we pointed out earlier, the effects of THF/HFF on semiconductor laser modulation have been studied using a linear gain model. ${ }^{3,5}$ According to the linear theory, a deeper modulation can always be achieved through further increase of THF/HHF amplitude. Since certain level of depth is required in application, it is important to know at what density/temperature level, the linear gain model will become invalid.

First of all, let us consider the nonlinear effect of application of an intense $\mathrm{THz}$ field on the differential gains induced by density change and temperature change. As understood from small-signal analysis in direct current-injection modulations, the important bandwidth parameter, $f_{3 d B}$, is determined partly by the density-induced differential gain. When the optical gain modulation is realized via plasma heating, it is found that the temperatureinduced differential gain replaces its density counterpart. However, deviation from linearized model is expected. The optical gain $G$ of the inverted medium, i.e., the EHP, is given as below,

$$
G=-\frac{v_{g} K \Gamma}{\epsilon_{0} \epsilon_{b}} \Im\left\{\frac{2}{V} \sum_{\bar{k}} d_{c v}(\vec{k}) \chi_{\vec{k}}^{*}\right\}
$$

In Fig. 1 (a) and (b), we plot the density- and temperature-induced differential gains $\{\partial G / \partial N, \partial G / \partial T\}$, respectively. It is obvious from the figure that these differential gains change appreciably within the relevant density and temperature ranges (see Fig. 2 and 3 ). Such gain nonlinearities can be understood as follows. Because phase space filling offect of the formionic nature of electrons and holes, saturation in the optical gain sets in as carrier density increases, which explains why the density-induced differential gain $\partial G / \partial V$ is reduced 
to zero. Plasma heating, however, reduces the phase filling factor, $f_{e}(\vec{k})+f_{h}(\vec{k})-1$, and thus cases the saturation and leads to a slight increase in $\partial G / \partial N$. On the contrary, as plasma heating reduces the phase filling factor, the temperature-induced differential gain $\partial G / \partial T$, proportional to the factor, decreases accordingly. As carrier density increases, saturation in the optical gain due to phase space filling suppresses the differential gain too.

Now let us take a look at the asymptotic (long-time) behavior of the system under a CW THF. According to our extensive simulation in a quite wide range of modulation parameters, the asymptotic behavior of laser intensity, the EHP density and temperature, and optical gain are all well characterized as the sum of a zero-frequency (DC) component and a second harmonic $\left(2 \nu_{T H z} \mathrm{AC}\right)$ component. Time evolution of these quantities start to deviate from this simple sinusoidal behavior when DC level of the laser output approaches zero-laser driven close to switch-off, or when the modulation frequency becomes too low and nearly resonant with the relaxation oscillation frequency. ${ }^{5}$ This sinusoidal behavior with the second harmonic oscillation has its origin in the $F_{T H z}^{2}(t)$ term in Eq. (2.20). The time evolution of laser intensity and carrier density can be found in Ref. 5. To look more closely at the modulation behavior as THF intensity changes, we plot the DC components (Fig. 2) and AC components (Fig. 3) of EHP temperature, EHP density, and laser intensity. There are quite a few features in Figs. 2 and 3 worth noting. First, all the DC components change with THF intensity linearly. As THF intensity increases, the DC plasma temperature rises due to heating effect. This temperature rise causes reduction in the optical gain. To tend to compensate for the loss of the optical gain due to temperature rise and to maintain laser operation, the EHP density has to increase to increase gain. This results in a net gain loss which cause a linear decrease of the laser output power as can be seen in Fig 2 (c). Finally, at the high level of the THF of our simulation, the gain loss due to the rising temperature far exceds the density-induced increase, the net gain becomes comparable to threshold level and laser output drops to almost zero. The second feature of Fig. 2 is the frequency insensitivity of the DC components. As is obvions from the figure, the DC components for 
all four frequencies, $0.1,0.33,0.66$ and ITHz fall on the same straight lines. This is because the inverse of the characteristic lynamical time scale in the EHP is at least an order of magnitude larger than these frequencies, thus the frequency dependence of the effective relaxation time $\tau_{T H z}$ becomes negligible, as can be seen in Eq. (2.16).

In contrast to the behavior of the $\mathrm{DC}$ components, the $\mathrm{AC}$ part shows a sensitive frequency dependence, as shown in Fig. 3. The AC components decrease as modulation frequency increases. This is a quite important point. Since a minimum modulation depth is required for application, this result places an upper limit to the modulation frequency. The most important feature in the $\mathrm{AC}$ component curves is the decrease of the $\mathrm{AC}$ components of density and laser intensity at high field amplitude (Fig. 3 (b) and (c)). While AC temperature shows a linear increase with THF intensity in the entire simulation range shown in the figure, the $\mathrm{AC}$ density and laser intensity increase only up to a particular THF amplitude when it reaches the maxima. Thereafter, the $\mathrm{AC}$ density and laser intensity start to drop. With further increase of the THF, the modulation depth approaches zero. This is contrary to the prediction of linear theory.

To explain this behavior, we go back again to the quantity of optical gain. The total gain variation is written as the sum of two terms: $\Delta G=\Delta G_{N}+\Delta G_{T}$ with $\Delta G_{N}=\partial G / \partial N \times \Delta N$ and $\Delta G_{T}=\partial G / \partial T \times \Delta T$. Typically in our simulation results, the $\mathrm{AC}$ component of density is in the order of $\Delta N=10^{8} \mathrm{~cm}^{-2}$ (see Fig. 3 (b)), while that of temperature is in the order of ten degrees (see Fig. 3 (a)). Using the typical number of differential gain in Fig. 1, we estimate that $\Delta G_{N}<0.1 \mathrm{~cm}^{-1}$ and $\Delta G_{T} \sim 1 \mathrm{~cm}^{-1}$. This means that the gain modulation due to temperature modulation is at least one order of magnitude larger that that due to density modulation. This is actually the reason why this modulation scheme can get around of the interband speed bottleneck, since no appreciable density change is involved. As a consequence, we ignore the gain change due to density modulation in the following discussions. We note that the depth of laser output modulation depends on the ability to modulate laser gain. It is therefore reasonable te assume that laser modulation elepth is proportional to the gain modulation. For a linear variation of gain with temperature, 
$\partial C / \partial T$ is constant. With the monotonous increase of $\mathrm{AC}$ temperature component as shown in Fig. 3 (a), a monotonous increase in AC laser intensity should be expected. The quite nonlinear behavior shown in Fig. 3 (c) indicates that the linear picture is no longer correct.

To look at this issue more quantitatively, we plot in Fig. 4, the temperature-induced gain change as a function of $\mathrm{AC}$ temperature component using the linear gain model and a nonlinear model up to second order in $\Delta T$. Since $A C$ temperature is proportional to the THF intensity (Fig. 3 (a)), the $x$-axis can be also understood as the THF intensity. The parameters are exactly the same as in Fig. 3 with the reference temperature being $295 \mathrm{~K}$. We see that, with the inclusion of gain nonlinearity, the gain modulation first increase up to a maximum and then starts to decrease as the nonlinearity starts to kick in. This explains why the $\mathrm{AC}$ laser intensity decreases as seen in Fig. 3 (c). This result has important consequence for application of this modulation scheme. Instead of simply increasing the THF intensity to increase modulation depth, a more careful optimization is required to achieve maximum modulation depth. This is especially important, since the relative modulation depth in this scheme is quite small very often. An estimate from Figs. 2 and 3 shows that the relative modulation depth is around $10^{-5}$.

Indeed, maximizing the modulation depth is an important task for the application of this modulation scheme. From our numerical results presented above, we can point out several directions to pursue for this maximization. One obvious direction is to decrease the modulation speed as the modulation depth increases inversely with frequency as can be seen in Fig. 3 (c). In fact, we have not been able to achieve a reasonable modulation depth for frequency beyond $1 \mathrm{THz}$ with $\mathrm{CW}$ THF of a few $\mathrm{KV} / \mathrm{cm}$. With careful optimization, this scheme may achieve decent depth of modulation in the range between a few tens of gigahertz $(\mathrm{GHz})$ to around $100 \mathrm{GHz}$. This is still a very appealing bandwidth, given the fact that the direct current modulation can achieve around $10 \mathrm{GHz}$. Another direction for maximization is to optimize the laser operating point, i.e., the operating threshold enrrent density ete. Plotted in Fig. 5are long-time simulated dynamies of the output intensity of a Gals QW haser operating at $79 K$ and under higher injertion levels, $l$ and $2 \mathrm{KAl} / \mathrm{cm}^{2}$. 
It is clearly indicated that modulation depth increases with the injection current. As a result, a decent $\sim 5 \%$ relative modulation depth can be achieved at high enough modulating THF intensity. It should be pointed out that the increase in modulation depth mainly depends on the injection level instead of the lattice temperature. From our analysis above, we understand that the temperature-induced gain nonlinearity is the major obstacle for achieving deep modulation. From Fig. 1 (b), we see that $\partial G / \partial T$ becomes a less sensitive function of temperature (meaning a smaller gain nonlinearity) as density increases. Increase of lasing threshold will have the laser operate around larger carrier density and therefore higher bandwidth. This can be seen in Fig. 4 by comparing the two curves for $N=1.0 \times$ $10^{12} \mathrm{~cm}^{-2}$ and $N=1.8 \times 10^{12} \mathrm{~cm}^{-2}$. Much less severe saturation of gain modulation is seen for the larger density case. Therefore increase in modulation depth is expected for the larger density. There is another possibility to maximize the modulation depth. We know that the gain characteristics depend on material properties and quantum well structures. By engineering quantum well structures with smaller gain nonlinearity, we may be able to increase modulation depth. Finally, modulation depth can be increased if pulsed $\mathrm{THz}$ field is used. This and other related issues will be discussed in detail in a forthcoming paper.

\section{CONCLUSIONS}

To conclude, we derived a set of moment equations from the microscopic semiconductor Bloch equations describing a semiconductor quantum well laser in an in-plane $\mathrm{THz}$ electric field. This self-consistent treatment of the lasing and heating processes in the presence of a heating field allows a better treatment of this system. As a result, we predict the strong saturation and degradation of moduation depth at relatively small $\mathrm{TH}_{z}$ intensity level. This places a severe limit to the bandwidth achievable using this scheme. The saturation phenomenon is fully understood using the nonlinear gain model, which we obtained automatically by starting from the microseopic thoory. Based on om understanding, wo are able to point ont sewral strategies for achieving deeper modulation. While this heating scheme 
proves not to be viable for frequency beyond $1 \mathrm{TH} z$, it provides an attractive alternative at $100 \mathrm{GHz}$ level or lower.

The key issue with this scheme that has to be resolved is how to apply the THF or HFF to a semiconductor laser. There are two ways to realize this scheme. Adding lateral electrode to bias the laser structure in the lateral direction provides an easy way as was suggested in Ref. 3. For demonstration purpose, terahertz wave can be coupled from the side of the laser structure into the active region. The mathematical treatment presented in this paper should be applicable for both cases.

Finally, we point out that semiconductor laser driven by a lateral heating field may have other application, such as switching, HFF detection, and short pulse generation etc. The detailed discussion of these issues will be beyond the scope of this paper and will be presented elsewhere.

\section{ACKNOWLEDGMENTS}

This work is supported by the Director's Discretionary Fund of the NASA Ames Research Center under NASA contract NAS2-14303.

\section{APPENDIX: MOMENTUM RELAXATION DUE TO ELECTRON-HOLE SCATTERINGS}

Carrier-carrier collisions occur on the femtosecond time scale at typical lasing plasma density. ${ }^{24,25}$ Intraband and interband scattering rates are comparable. To investigate the consequences of interband scatterings on momentum relaxation we assume quasiequilibrium for the electron-hole plasma.

Given that each subband is thermalized and described by a drifted Fermi-Dirac distri-

bution function, $n_{\vec{k}}^{\alpha} \equiv f_{r}^{(0)}\left(\vec{k}-\vec{k}_{l}^{(\alpha)}\right)$, where $\alpha=e, h$, the electron-hole scattering rate can be written as follows 


$$
\begin{aligned}
\left.\frac{\partial n_{\vec{k}}^{\alpha}}{\partial t}\right|_{e h}=\sum_{\vec{k}^{\prime}, \vec{q} \neq 0} \frac{2 \pi}{\hbar} l_{\vec{q}}^{2} \delta\left(\epsilon_{\vec{k}}^{\alpha}+\epsilon_{\vec{k}^{\prime}+\vec{q}}^{\beta}-\epsilon_{\vec{k}^{\prime}}^{\beta}-\epsilon_{\vec{k}+\vec{q}}^{\alpha}\right) & {\left[n_{\vec{k}+\vec{q}^{\prime}}^{\alpha} n_{\vec{k}^{\prime}}^{\beta}\left(1-n_{\vec{k}}^{\alpha}\right)\left(1-n_{\vec{k}^{\prime}+\vec{q}^{\prime}}^{\beta}\right)\right.} \\
& \left.-n_{\vec{k}}^{\alpha} n_{\vec{k}^{\prime}+i^{\prime}}^{\beta}\left(1-n_{\vec{k}^{\prime}}^{\beta}\right)\left(1-n_{\vec{k}+\vec{q}^{\prime}}^{\alpha}\right)\right],
\end{aligned}
$$

where $\alpha$ and $\beta$ differ, and $V_{\vec{q}}$ is the screened Coulomb potential. After factorization, the difference term in the squared bracket contains a factor

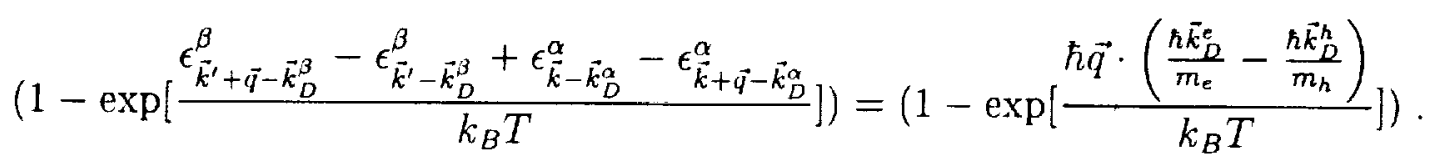

where we have used energy conservation and parabolicity of carrier energy dispersion. Expansion of the exponent term leads to the conclusion that

$$
\left.\frac{\partial n_{\vec{k}}^{e}}{\partial t}\right|_{e h} \equiv-\left.\frac{\partial n_{\vec{k}}^{h}}{\partial t}\right|_{e h} \propto \frac{\hbar \vec{k}_{D}^{e}}{m_{e}}-\frac{\hbar \vec{k}_{D}^{h}}{m_{h}}=\left(\frac{\hbar k_{D}^{e}}{m_{e}}-\frac{\hbar k_{D}^{h}}{m_{h}}\right) \hat{x}
$$

After integrating over $\vec{k}$ for the scattering terms above according to Eq. (2.11), and realizing that the following relationship holds under the assumption of quasiequilibrium,

$$
P_{\alpha}=N \hbar k_{D}^{\alpha}
$$

it is straightforward to show that the momentum relaxation due to electron-hole scatterings can be written in a rate equation form as in Eq. (2.15)

$$
\left.\frac{d P_{e}}{d t}\right|_{e h} \equiv-\left.\frac{d P_{h}}{d t}\right|_{e h} \approx-\gamma_{e h} m_{r}\left(\frac{P_{e}}{m_{e}}-\frac{P_{h}}{m_{h}^{\prime}}\right)
$$

and the relaxation rate is given by

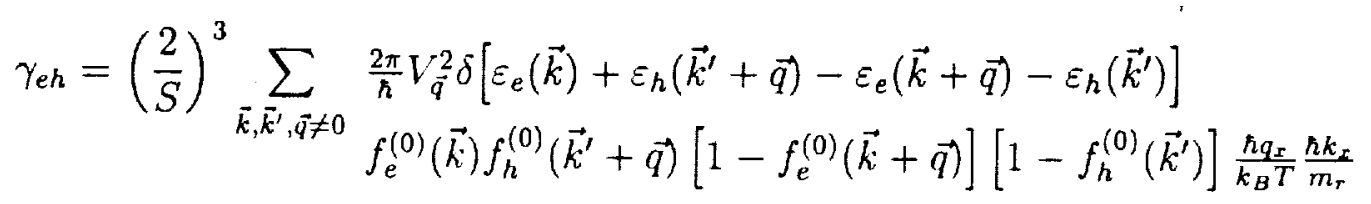

in the leading order of $\frac{\hbar k_{0}^{e}}{m_{e}}-\frac{\hbar k_{D}^{h}}{m_{h}}$,

Finally, we should comment on the use of the equality $T_{e}=T_{h}$ in deriving Eq. (A3). Exactly speaking, $T_{e}=T_{h}$ should be reached on the same time scale as in Eq. (A5). This means that the above derivation is not self-consistent on time scale of $\gamma_{e h}^{-1}$. Since we finally climinate $P_{e}$ and $P_{h}$ addiabatically using Eq. (2.16), our treaturent on long time scale is, however, self-consistent. 


\section{REFERENCES}

${ }^{1}$ At the time we are preparing for this manuscript, progress is being made in the more than 110 gigahertz organic modulator study. See Y. Shi, C. Zhang, H. Zhang, J. H. Bechtel, L. R. Dalton, B. H. Robinson, and W. H. Steier, Science 288, 119 (2000).

${ }^{2}$ V. B. Gorfinkel' and I. I. Filatov, Sov. Phys. Semicon. 24, 466 (1990).

${ }^{3}$ S. A. Gurevich, I. I. Filatov, B. M. Gorbovitsky, and V. B. Gorfinkel', Proc. AIP for Joint Sov.-Am. Workshop on the Phys. of Semicon. Lasers, 67 (1992).

${ }^{4}$ S. Hughes and D. S. Citrin, Phys. Rev. B 58, R15969 (1998).

${ }^{5}$ C. Z. Ning, S. Hughes, and D. S. Citrin, Appl. Phys. Lett. 75, 442 (1999).

${ }^{6}$ G. P. Agrawal, Appl. Phys. Lett. 57, 1 (1990).

${ }^{7}$ M. Lindberg and S. W. Koch, Phys. Rev. B 38, 3342 (1988).

${ }^{8}$ H. Haug and S. W. Koch, Phys. Rev. A 39, 1887 (1989).

${ }^{9}$ S. W. Koch and H. Haug, Theory of the Electrical and Optical Properties of Semiconductors (World Scientific, Singapore, 1992).

${ }^{10}$ S. W. Koch, T. Meier, J. Hader, K.-C. Je, F. Rossi, and P. Thomas, in Frontiers in nanoscale science of micron/submicron devices, edited by A.-P. Jauho and E. V. Buzaneva (Kluwer Academic, Dordrecht, 1996), p.459.

${ }^{11}$ T. Meier, G. von Plessen, P. Thomas, and S. W. Koch, Phys. Rev. Lett. 73, 902 (1994).

${ }^{12}$ T. Meier, G. von Plessen, P. Thomas, and S. W. Koch, Phys. Rev. B 51, 14490 (1995).

${ }^{13}$ L. P. Kadanoff and G. Baym. Quantum Statistical Mechanics (Addison-Wesley, Reading, 1989).

14. Hess and T. Kuhn, Phys. Rer A 54, 3347 (1996).

${ }^{15}$ C. Z. Ning, R. A. Indik, J. V. Moloney, W. W. Chow, A. Girndt, S. W. Koch, and R. 
Binder, in Physics and Simulation of Optoelectronic: Devices V, cdited by M. Osinski and W. W. Chow, SPIE Proc. No. 2994 (SPIE, Bellingham, 1997), p. 666.

${ }^{16}$ C. Z. Ning, R. A. Indik, and J. V. Moloney, J. Opt. Soc. Am. B 12, 1993 (1995).

${ }^{17}$ C. Z. Ning, R. A. Indik, J. V. Moloney, and S. W. Koch, in Physics and Simulation of Optoelectronic Devices III, edited by M. Osinski and W. W. Chow, SPIE Proc. No. 2399 (SPIE, Bellingham, 1995), p. 617.

${ }^{18}$ C. Z. Ning, S. Bischoff, S. W. Koch, G. K Harkness, J. V. Moloney, and W. W. Chow, Optical Engineering, 37, 1175 (1998).

${ }^{19}$ M. Lundstrom, Fundamentals of Carrier Transport (Modular Series on Solid State Devices, Addison-Wesley, 1990).

${ }^{20}$ W. W. Chow and S. W. Koch, Semiconductor-Laser Fundamentals - Physics of the Gain Materials (Springer, Berlin, 1999).

${ }^{21}$ W. W. Chow, G. C. Dente, and D. Depatie, IEEE J. Quantum Electron. QE-23, 1314 (1987).

${ }^{22}$ B. K. Ridley, Electrons and Phonons in Semiconductor Multilayers (Cambridge University Press, Cambridge, 1997).

${ }^{23}$ H. Rücker, E. Molinari, and P. Lugli, Phys. Rev. B 44, 3463 (1991):

${ }^{24}$ R. Binder, D. Scott, A. E. Paul, M. Lindberg, K. Henneberger, and S. W. Koch, Phys. Rev. B 45, 1107 (1992).

${ }^{25}$ S. Bolton, G. Sucha, D. Chemla, D. L. Sivco, and A. Y. Cho, Phys. Rev. B 58, 16326 (1998).

${ }^{26}$ C. Z. Ning, R. A. Indik, and J. V. Moloney, IEEE J. Quan. Electron. 33, 1543 (1997).

27 J. Li and C. Z. Ning, unpublished. 


\section{TABLES}

TABLE I. Partial Simulation Parameters

\begin{tabular}{|c|c|c|c|c|c|}
\hline$\Gamma$ & confinement factor & 0.025 & $\tau_{p h}$ & photon lifetime & $40 \mathrm{ps}$ \\
\hline$J_{i n j}$ & injection current & $0.4 \mathrm{KA} / \mathrm{cm}^{2}$ & $\eta$ & current inj. efficiency & 0.5 \\
\hline$B_{s p}$ & $\begin{array}{c}\text { spontaneous emission } \\
\text { coefficient }\end{array}$ & $\begin{array}{c}4.0 \times 10^{-10} \\
\mathrm{~cm}^{3} / \mathrm{s}\end{array}$ & $\gamma_{n r}$ & $\begin{array}{c}\text { non-radiative recomb. } \\
\text { coefficient }\end{array}$ & $\begin{array}{c}1.0 \times 10^{9} \\
s^{-1}\end{array}$ \\
\hline$\tau_{T H z}$ & relaxation time & $0.1 p s$ & $\hbar \gamma_{p}$ & dephasing rate & $5 \mathrm{meV}$ \\
\hline
\end{tabular}




\section{APPENDIX: FIGURE CAPTIONS}

Fig. 1: Differential gains versus temperature at various densities. Density-induced differential gain and temperature-induced differential gain are plotted in (a) and (b), respectively.

Fig. 2: Dependence of DC components of long-time solutions for plasma temperature (a), density (b), and laser intensity (c) on THz field intensity at $0.1,0.33,0.66$, and $1.0 \mathrm{THz}$.

Fig. 3: Dependence of modulation amplitudes ( $2 \nu_{T H z}$ component) of plasma temperature (a), density (b), and laser intensity (c) on $\mathrm{THz}$ field intensity at $0.1,0.33,0.66$, and $1.0 \mathrm{THz}$.

Fig. 4: Temperature-induced nonlinearity in optical gain. Plotted is the change in gain due to plasma heating, $\Delta G=\partial G /\left.\partial T\right|_{T_{r}} \times \Delta T+\partial^{2} G /\left.\partial T^{2}\right|_{T_{r}} \times(\Delta T)^{2} / 2$, where reference temperature $T_{r}$ is $295 \mathrm{~K}$, at two plasma densities. Dashed-line data only keep the linear term.

Fig. 5: Long-time behavior of laser output operating at $77 K$ and under $\mathrm{THz}$ field modulation. Injection current for the dotted curves is $2 \mathrm{KA} / \mathrm{cm}^{2}$ and $1 \mathrm{KA} / \mathrm{cm}^{2}$ for the solid curves. 


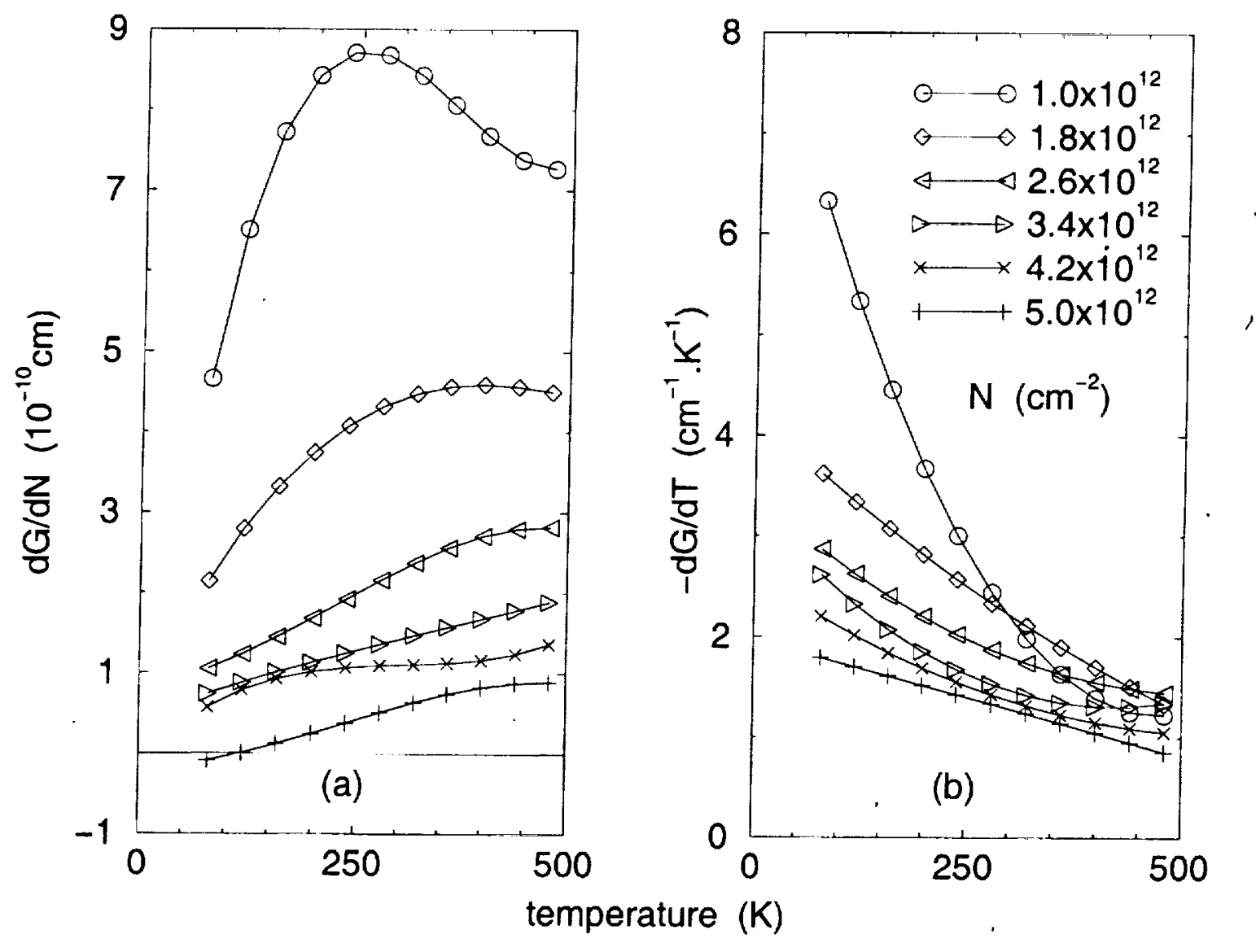

Fig. 1/Jianzhong Li/J. Appl. Phys. 

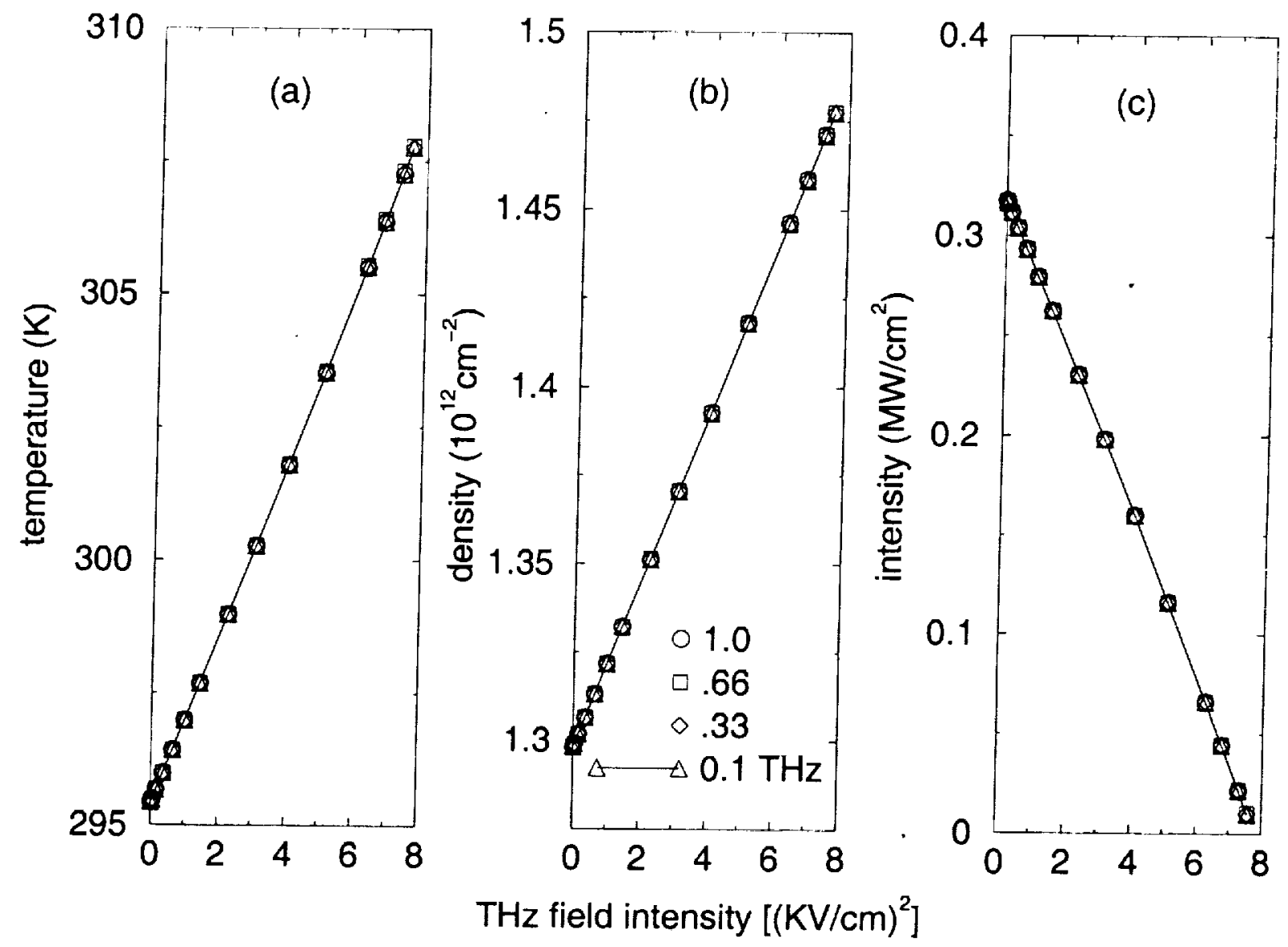

Fig. 2/Jianzhong Li/J. Appl. Phys. 

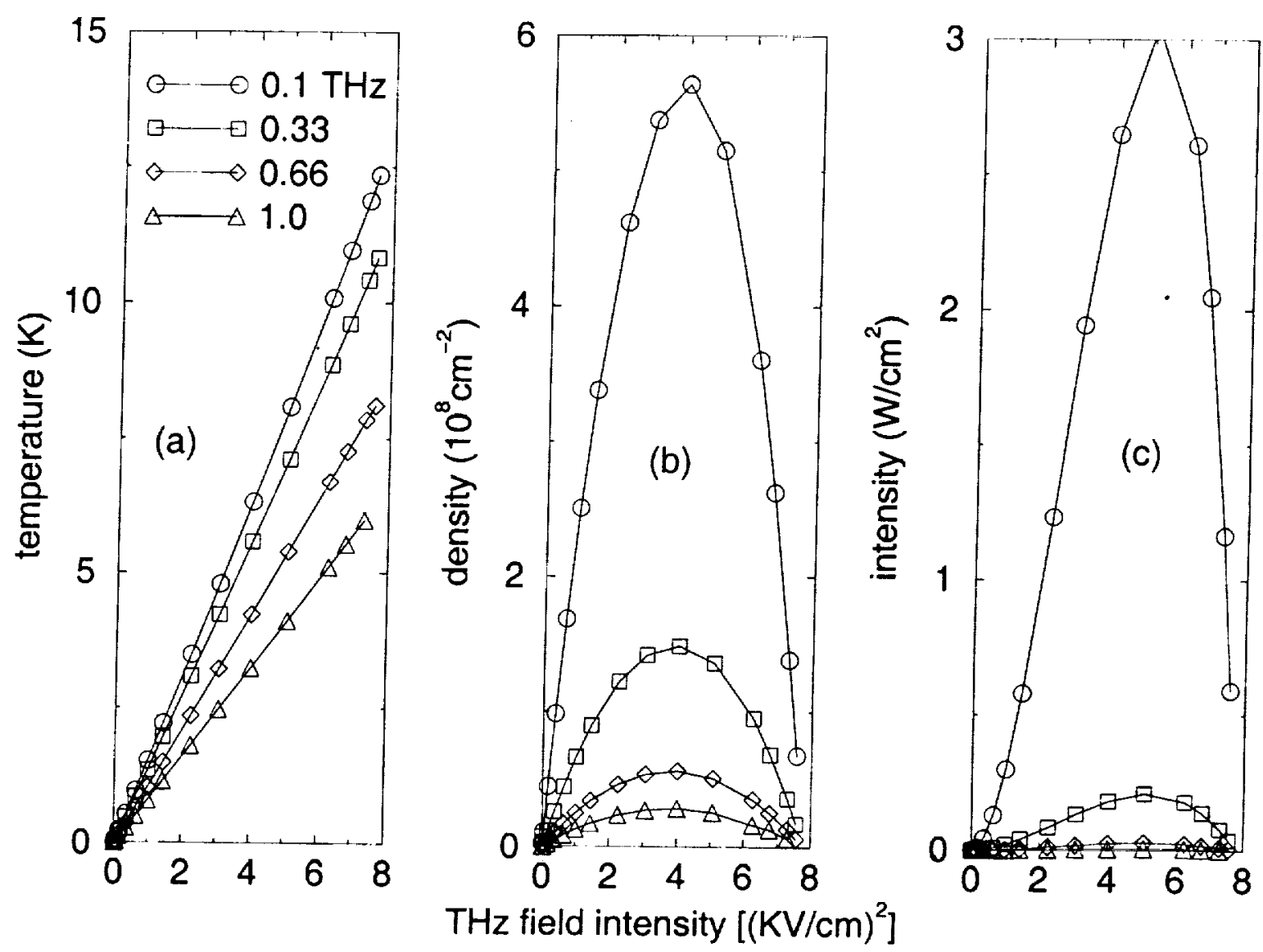

Fig. 3/Jianzhong Li/J. Appl. Phys. 


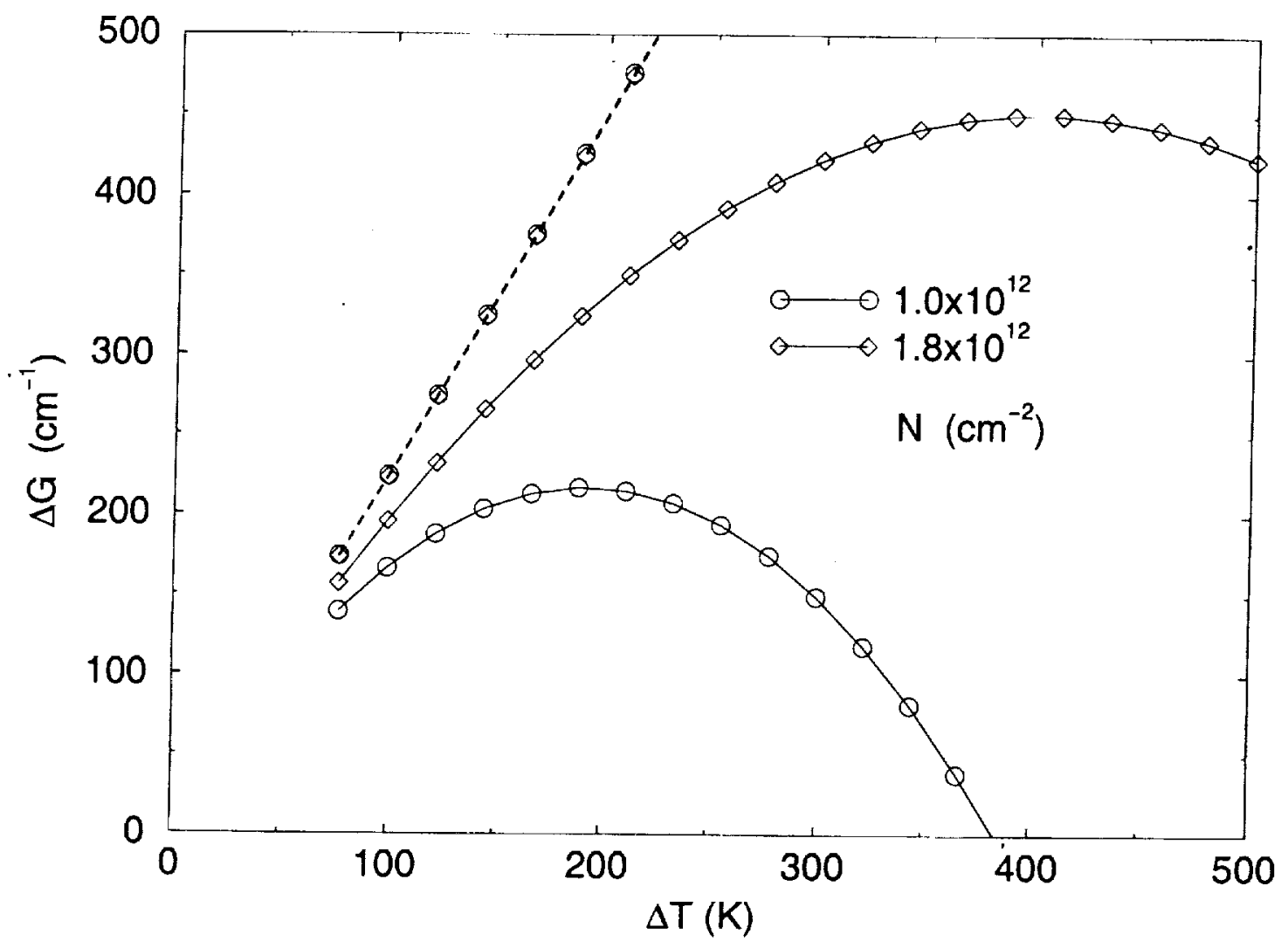

Fig. 4/Jianzhong Li/J. Appl. Phys. 


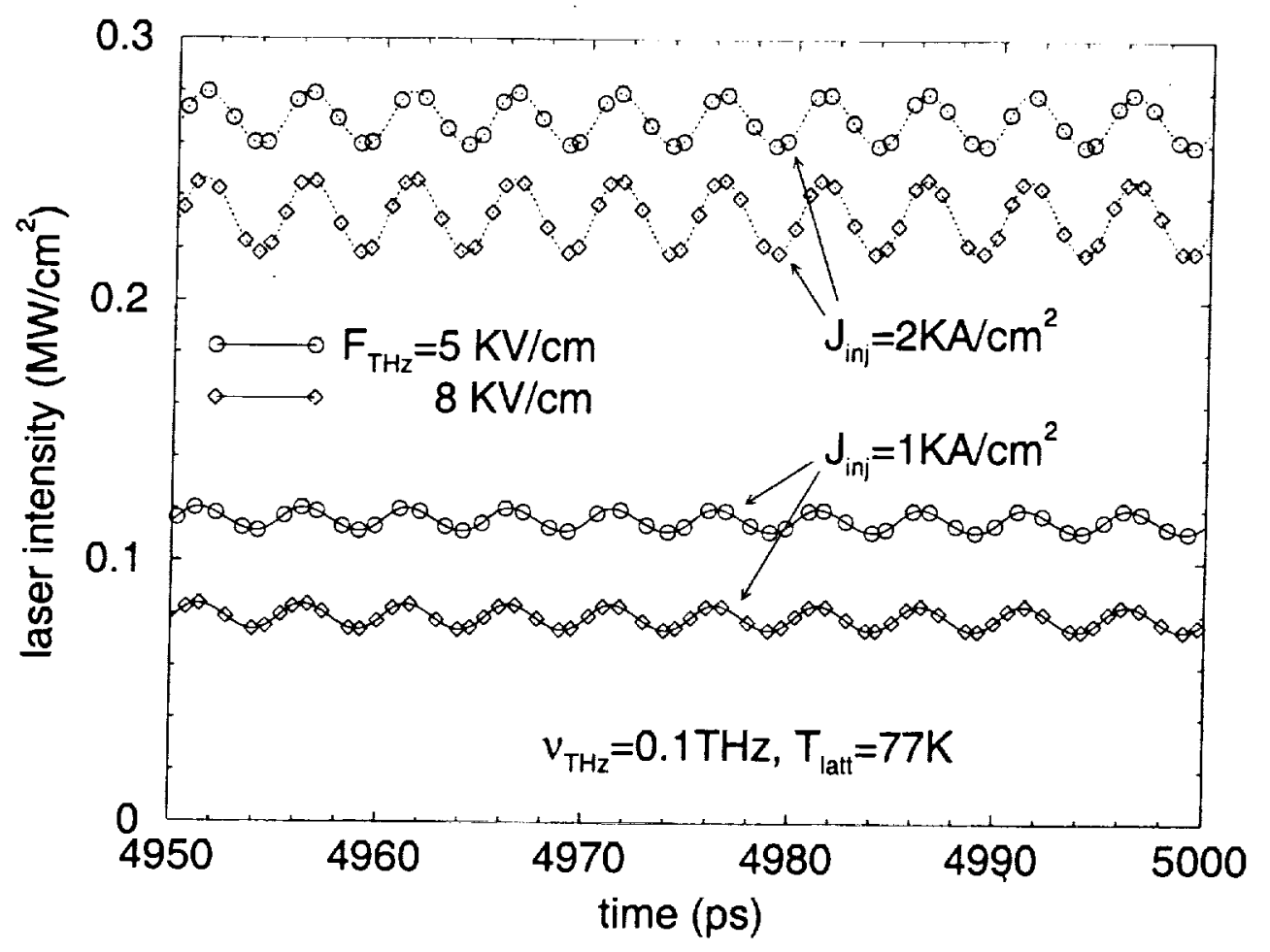

Fig. 5/Jianzhong Li/J. Appl. Phys. 